\title{
TENDÊNCIAS ANATÔMICAS NA FLORA SUL-RIO-GRANDENSE. 1 - ELEMENTOS VASCULARES ${ }^{1}$
}

\author{
SIDINEI RODRIGUES DOS SANTOS ${ }^{2}$ JOSE NEWTON CARDOSO MARCHIORI ${ }^{3}$
}

\section{RESUMO}

O presente estudo objetivou reunir informações e estabelecer tendências sobre a anatomia da madeira de espécies nativas no Rio Grande do Sul. Os dados foram extraídos, basicamente, de artigos publicados pelos autores entre 1980 e 2009. Foram investigadas 79 espécies, de 24 famílias de Dicotiledôneas. Anéis de crescimento distintos, placas de perfuração simples e pontoações intervasculares alternas aparecem em mais de $90 \%$ das espécies investigadas. São também predominantes: porosidade difusa; poros muito pequenos $(<50 \mu \mathrm{m})$, agrupados em múltiplos, em arranjo radial/racemiforme e com densidade superior a $20 / \mathrm{mm}^{2}$; elementos vasculares curtos $(<350 \mu \mathrm{m})$; e pontoações intervasculares pequenas $(4-7 \mu \mathrm{m})$, não ornamentadas. Os padrões observados podem ser explicados, em parte, pelo elevado número de espécies de determinadas famílias botânicas, bem como pelo posicionamento latitudinal do Estado.

Palavras-chave: Anatomia da madeira, elementos vasculares, anéis de crescimento.

\section{ABSTRACT}

[Anatomical trends in the flora of Rio Grande do Sul state. 1 - Vascular elements].

The present work aimed to gather information and to establish wood anatomical trends in native species from Rio Grande do Sul state, Brazil. The analyzed data were obtained, basically, from papers published by the authors, between 1980 and 2009. Seventy nine species were investigated, belonging to 24 families of Dicotyledons. Distinct growth rings, simple perforation plates and alternate intervessel pits appear in more than $90 \%$ of the species. The following features are also predominant: diffuse porosity; pores in multiples, organized in radial/racemiform pattern; very small pores $(<50 \mu \mathrm{m})$, more numerous than $20 / \mathrm{mm}^{2}$; short vessel elements $(350 \mu \mathrm{m})$; and non-vestured intervessel pits $(4-7 \mu \mathrm{m})$. The results may be partly attributed to the great number of species belonging to certain botanical families and to the latitudinal position of Rio Grande do Sul state.

Key words: Wood anatomy, vessels, growth rings.

\section{INTRODUÇÃO}

A anatomia da madeira, ao estudar a estrutura interna do xilema secundário das plantas lenhosas, proporciona uma apreensão empírica do comportamento tecnológico e das possíveis utilizações da madeira (Teixeira, 1977), além

1 Recebido para publicação em 20-I-2010 e aceito para publicação em 15-III-2010.

2 Biólogo, bolsista (CNPq- Brasil), doutorando do Programa de Pós-Graduação em Engenharia Florestal, Departamento de Ciências Florestais, Universidade Federal de Santa Maria. CEP 97105-900. Santa Maria, RS, Brasil. sthurt.bio@gmail.com

${ }^{3}$ Engenheiro Florestal, Dr., bolsista de Produtividade em Pesquisa (CNPq - Brasil). Prof. Titular do Departamento de Ciências Florestais, Universidade Federal de Santa Maria, Santa Maria, RS, Brasil. balduinia@mail.ufsm.br de auxiliar na identificação de espécies, gêneros e famílias botânicas (Marchiori \& OliveiraDeble, 2007; Santos \& Marchiori, 2009a), bem como em investigações taxonômicas, filogenéticas e evolutivas (Wheeler \& Baas, 1991; Schirarend, 1991; Herendeen et al., 1999).

Nas últimas décadas, observa-se uma ênfase crescente em pesquisas direcionadas à avaliação da influência do ambiente na estrutura anatômica, sobretudo pela comparação de floras de regiões distintas, como nos estudos de Baas (1973), Baas et al. (1983), Barajas-Morales (1985), Carlquist \& Hoekman (1985), Baas \& Schweingruber (1987), Wheeler \& Baas (1991), Lindorf (1994) e León (2005). Como salientado por Marchiori (1983a), o resultado destas pesquisas auxilia na compreensão do compor- 
tamento do xilema em condições ambientais diversas, fornecendo subsídios valiosos para a interpretação do crescimento das plantas, além das possibilidades anteriormente referidas.

Apesar da importância, são ainda escassos os trabalhos sobre a anatomia de madeiras no Brasil, destacando-se, neste aspecto, as contribuições de Mainieri \& Chimelo (1989), Alves \& Angyalossy-Alfonso (2000; 2002), Cury (2002), Mattos et al. (2003) e Barros et al. (2006). No Rio Grande do Sul, não chega a 100 o número de espécies investigadas sob o ponto de vista da estrutura anatômica, sendo que, somente de árvores, foram listadas mais de 500 espécies para o Estado (Reitz et al., 1983). Além de escassas, as referências sobre madeiras sulrio-grandenses são fragmentárias e geralmente restritas a espécies isoladas.

O presente estudo visa a reunir informações anatômicas, bem como reconhecer tendências estruturais de madeiras nativas na flora sul-riograndense.

\section{MATERIAIS E MÉTODOS}

Situado no extremo sul do Brasil, em zona extratropical, entre os paralelos $27^{\circ} 04^{\prime} 49^{\prime \prime}$ e $33^{\circ} 45^{\prime} 00^{\prime \prime} \mathrm{S}$ e os meridianos $49^{\circ} 42^{\prime} 22^{\prime \prime}$ e 57 38’34" O, o Rio Grande do Sul tem clima predominantemente úmido, com precipitação média de 1539 (1162-2162) $\mathrm{mm}$ e temperatura média anual de $17,9{ }^{\circ} \mathrm{C}$ (Maluf, 2000). As chuvas, variáveis durante o ano, geralmente superam a evapotranspiração, havendo, todavia, forte sazonalidade na temperatura, entre inverno e verão (Brasil, 1973). As formações vegetacionais do Estado incluem Floresta Densa, Floresta Estacional Decidual, Floresta Estacional Semidecidual e Floresta Ombrófila Mista, além de capões-de-mato e matas ciliares (Marchiori, 2002).

No presente estudo, são analisadas 79 espécies, entre árvores, arbustos e lianas, distribuídas em 24 famílias de Dicotiledôneas, sendo os resultados confrontados com referências da literatura sobre espécies brasileiras e de outras partes do Mundo.
Os dados anatômicos, resumidos na Tabela 1 , foram extraídos, basicamente, de artigos publicados pelos autores no período de 1980 a 2009, nos seguintes periódicos: Revista do Centro de Ciências Rurais, Ciência e Natura, Ciência Florestal, Ciência Rural e Balduinia. As espécies são apresentadas por ordem alfabética de família e nomes científicos. As referências bibliográficas (RB) relativas às distintas espécies são indicadas no alto da página 6 , bem como na lista da bibliografia consultada, ao final do texto.

Foram avaliados os seguintes aspectos: presença de anéis de crescimento; porosidade; comprimento e diâmetro de elementos vasculares; arranjo/agrupamento e frequiência de poros; tipo de placa de perfuração; presença de espessamentos espiralados; tamanho e arranjo de pontoações intervasculares. $\mathrm{Na}$ análise das características quantitativas foram utilizados os valores médios de cada caráter, agrupados em intervalos, conforme as categorias estabelecidas pelo IAWA Committee (1989). As características qualitativas seguem o descrito pelos respectivos trabalhos. No caso do arranjo e agrupamento de poros foram estabelecidas as seguintes categorias, com vistas a abranger os padrões relacionados: 1 - poros solitários/sem padrão definido de organização; 2 - poros em múltiplos radiais/racemiformes; 3 - poros em padrão diagonal/dendrítico.

\section{RESULTADOS E DISCUSSÃO}

\section{Anéis de crescimento}

A presença de anéis de crescimento foi observada em 72 das 79 espécies estudadas, número que representa, aproximadamente, $91 \%$ do total. Alves \& Angyalossy-Alfonso (2000), em estudo de tendências anatômicas na flora brasileira, assinalam este caráter para $48 \%$ das espécies, concentradas, notadamente, nas regiões sul e sudeste do país. Resultados semelhantes foram obtidos por Callado et al. (2001) e Marcati et al. (2006), comprovando o 
predomínio de espécies com anéis de crescimento distintos nas referidas regiões, em diferentes tipologias florestais.

A presença de anéis de crescimento é usualmente atribuída à sazonalidade climática, sobretudo à disponibilidade hídrica e à temperatura (Gomez \& Muñiz, 1986; Silva et al., 1991; Alves \& Angyalossy-Alfonso, 2000; Lisi et al., 2008). Em ambientes com alterações periódicas de qualquer um destes fatores, as espécies tendem a desenvolver camadas de crescimento, ao passo que em condições mais homogêneas, a atividade cambial mostra-se contínua. Os resultados do presente estudo contribuem favoravelmente a esta interpretação, pois o clima do Rio Grande do Sul caracterizase por forte oscilação na temperatura, entre inverno e verão. Cabe salientar, todavia, que qualquer fator que influi na atividade cambial pode induzir à formação de anéis de crescimento: é o caso, por exemplo, da ocorrência de inundações periódicas e da deciduidade foliar (Worbes, 1989; Callado et al., 2001), cujos efeitos assemelham-se aos causados pela seca ou baixas temperaturas. Fatores ambientais, entretanto, nem sempre são determinantes para a formação de anéis de crescimento. Em certos grupos taxonômicos, caso do gênero Guarea (Meliaceae), os anéis são indiferenciados, inclusive em espécies de clima estacional (Guarea macrophylla por exemplo).

\section{Porosidade}

A maior parte das espécies analisadas apresenta porosidade difusa $(78 \%)$ ou semidifusa (19\%), sendo que a porosidade em anel foi observada em apenas 3\% das espécies. O predomínio do padrão difuso está de acordo com a tendência geral verificada em Dicotiledôneas (Metcalfe \& Chalk, 1972; Wheeler et al., 2007) e, inclusive, em espécies brasileiras (Alves \& Angyalossy-Alfonso, 2000; Mattos et al., 2003; Barros et al., 2003; 2006).

Embora predominante na flora sul-riograndense, o percentual de espécies com porosidade difusa ficou abaixo do observado em regiões brasileiras de menor latitude (Mattos et al., 2003; Barros et al., 2003; 2006). Alves \& Angyalossy-Alfonso (2000) referem uma concentração maior de espécies com desvios da porosidade difusa em latitudes mais elevadas e/ ou ambientes com variação sazonal de temperatura, tendência corroborada no presente estudo. Há sempre que considerar, todavia, a influência da disponibilidade hídrica, uma vez que este aspecto pode ser determinante na distribuição dos poros na madeira. Woodcock (1994), entre outros, refere uma freqüência maior de espécies com redução gradual no diâmetro de poros em latitudes médias, variando a percentagem inversamente à precipitação.

\section{Agrupamento e arranjo de poros}

Das 79 espécies investigadas, 62 apresentaram poros em múltiplos, número correspondente a $78 \%$ do total. Alves \& AngyalossyAlfonso (2000) encontraram correlação positiva entre o referido caráter e a região sudeste do Brasil, e relação negativa com a região norte, postulando a existência de ligação entre agrupamento de poros e latitudes mais elevadas e/ou ambientes sazonais. Os resultados do presente estudo contribuem favoravelmente a esta interpretação, tendo em vista o posicionamento geográfico do Rio Grande do Sul. O predomínio de espécies com vasos em múltiplos foi também observado em floras do centro-oeste e sudeste brasileiro com clima sazonal, reforçando ainda mais tal perspectiva (Mattos et al., 2003; Barros et al., 2006). A ocorrência deste padrão de agrupamento de vasos tem sido relacionada, por muitos autores, a ambientes secos (Carlquist, 1984; Lindorf, 1994; León, 2005); no Rio Grande do Sul, todavia, o clima é nitidamente ombrófilo. Mesmo assim, esta condição pode resultar de um clima pretérito mais seco ou, como sugerem Alves \& Angyalossy-Alfonso (2000), ser uma resposta a fatores estressantes, caso da elevada variação da temperatura no Estado, ao longo do ano.

No tocante ao arranjo, observou-se que a maioria das espécies (cerca de 66\%) apresenta 
TABELA 1. Resumo das principais características anatômicas.

\begin{tabular}{|c|c|c|c|c|c|c|c|c|c|c|c|c|c|}
\hline Família / Espécie & RB & $\mathbf{H}$ & $\mathbf{P P}$ & $\mathbf{E E}$ & API & OPI & $\mathbf{A C}$ & $\mathbf{P}$ & $\mathbf{A P}$ & Fr & D & CEV & DPI \\
\hline \multicolumn{14}{|l|}{ Apocynaceae } \\
\hline Aspidosperma quebrachoblanco & 1 & 2 & s & - & a & - & + & 1 & 1 & 29 & 57 & 360 & 3,9 \\
\hline \multicolumn{14}{|l|}{ Asteraceae } \\
\hline Baccharis dracunculifolia & 2 & 1 & s & + & a & - & + & 1 & 3 & nd & 28 & 202 & 4,0 \\
\hline Baccharis longoattenuata & 3 & 1 & $\mathrm{~s}$ & + & a & - & + & 1 & 3 & nd & 32 & 230 & nd \\
\hline Baccharis milleflora & 3 & 1 & $\mathrm{~s}$ & - & a & - & + & 1 & 2 & nd & 27 & 204 & nd \\
\hline Baccharis patens & 2 & 1 & $\mathrm{~s}$ & + & a & - & - & 1 & 3 & nd & 25 & 143 & 5,0 \\
\hline Baccharis tridentata & 2 & 1 & $\mathrm{~s}$ & + & a & - & - & 1 & 2 & nd & 36 & 198 & 4,0 \\
\hline Heterothalamulopsis wagenitzii & 4 & 1 & $\mathrm{~s}$ & + & a & - & - & 1 & 3 & 301 & 23 & 178 & 5,0 \\
\hline Heterothalamus alienus & 5 & 1 & $\mathrm{~s}$ & + & a & - & - & 1 & 3 & 478 & 35 & 154 & 5,7 \\
\hline Heterothalamus psiadioides & 6 & 1 & s & + & $\mathrm{a}$ & - & - & 1 & 3 & 259 & 35 & 209 & 5,7 \\
\hline Heterothalamus rupestris & 5 & 1 & s & - & a & - & - & 1 & 3 & 363 & 32 & 162 & 5,0 \\
\hline \multicolumn{14}{|l|}{ Berberidaceae } \\
\hline Berberis laurina & 7 & 1 & s & + & a & - & + & 2 & 3 & 615 & 21 & 229 & 5,4 \\
\hline \multicolumn{14}{|l|}{ Bignoniaceae } \\
\hline Handroanthus pulcherrimus & 8 & 2 & $\mathrm{~s}$ & - & a & - & + & 1 & 2 & 14 & 56 & 258 & 9,5 \\
\hline \multicolumn{14}{|l|}{ Combretacee } \\
\hline Terminalia australis & 9 & 1,2 & s & - & a & + & + & 1 & 2 & 54 & 62 & 366 & 6,5 \\
\hline \multicolumn{14}{|l|}{ Ebenaceae } \\
\hline Diospyros inconstans & 10 & 2 & $\mathbf{s}$ & - & a & - & + & 1 & 2 & nd & 58 & 392 & 4,3 \\
\hline \multicolumn{14}{|l|}{ Escalloniaceae } \\
\hline Escallonia megapotamica & 11 & 1,2 & $\mathrm{~m}$ & + & 0 & - & + & 1 & 1 & 377 & 32 & 580 & 8,2 \\
\hline \multicolumn{14}{|l|}{ Euphorbiaceae } \\
\hline Sebastiana schottiana & 12 & 1 & $\mathbf{s}$ & - & a & - & + & 1 & 2 & 75 & 48 & 474 & 5,8 \\
\hline \multicolumn{14}{|l|}{ Fabaceae } \\
\hline Acacia bonariensis & 13 & 1 & $\mathrm{~s}$ & - & a & + & + & 2 & 2 & 20 & 118 & 238 & 6,5 \\
\hline Acacia caven & 14 & 2 & $\mathbf{s}$ & - & a & + & + & 1 & 2 & 14 & 92 & 167 & 6,6 \\
\hline Acacia ibirocayensis & 15 & 1 & $\mathbf{s}$ & - & a & + & + & 1 & 2 & 121 & 52 & 158 & 6,5 \\
\hline Acacia nitidifolia & 16 & 1 & $\mathrm{~s}$ & - & a & + & + & 1 & 2 & 27 & 130 & 374 & 8,8 \\
\hline Acacia recurva & 17 & 3 & $\mathbf{s}$ & - & a & + & + & 2 & 2 & 12 & 91 & 295 & 7,4 \\
\hline Acacia tucumanensis & 18 & 1,2 & s & - & a & + & + & 2 & 2 & 22 & 151 & 241 & 8,1 \\
\hline Albizia edwallii & 19 & 2 & $\mathrm{~s}$ & - & a & + & + & 1 & 2 & 3 & 143 & 247 & 8,3 \\
\hline Indigofera suffruticosa & 20 & 1 & $\mathbf{s}$ & - & a & + & + & 1 & 2 & 35 & 61 & 162 & 6,8 \\
\hline Lonchocarpus campestris & 21 & 1,2 & s & - & $\mathrm{a}$ & + & + & 1 & 2 & 11 & 84 & 189 & 9,1 \\
\hline Lonchocarpus nitidus & 22 & 2 & s & - & $\mathrm{a}$ & + & + & 2 & 2 & 10 & 156 & 195 & 7,8 \\
\hline Mimosa berroi & 23 & 1 & $\mathbf{s}$ & - & a & + & + & 1 & 2 & 53 & 41 & 197 & 4,8 \\
\hline Mimosa bimucronata & 24 & 1,2 & $\mathbf{s}$ & - & $\mathrm{a}$ & + & + & 2 & 2 & 11 & 107 & 107 & 7,1 \\
\hline Mimosa cruenta & 25 & 1 & $\mathrm{~s}$ & + & $\mathrm{a}$ & + & + & 1 & 2 & nd & 45 & 234 & 6,5 \\
\hline Mimosa daleoides & 26 & 1 & $\mathbf{s}$ & - & $\mathrm{a}$ & + & + & 1 & 2 & 51 & 67 & 248 & nd \\
\hline Mimosa eriocarpa & 27 & 1 & $\mathbf{s}$ & - & $\mathrm{a}$ & + & + & 1 & 2 & 31 & 58 & 289 & 6,3 \\
\hline Mimosa incana & 28 & 1 & $\mathrm{~s}$ & - & $\mathrm{a}$ & + & + & 1 & 2 & 28 & 81 & 215 & 5,9 \\
\hline Mimosa pilulifera & 29 & 1 & s & - & a & + & + & 1 & 2 & 29 & 70 & 221 & 7,5 \\
\hline Mimosa scabrella & 30 & 2 & s & - & $\mathrm{a}$ & + & + & 1 & 2 & 10 & 155 & 351 & 8,3 \\
\hline Mimosa sparsa & 31 & 1 & $\mathrm{~s}$ & - & $\mathrm{a}$ & + & + & 1 & 2 & 22 & 59 & 158 & 6,3 \\
\hline Mimosa trachycarpa & 32 & 1 & s & + & a & + & + & 1 & 2 & nd & 73 & 132 & 6,0 \\
\hline Mimosa uraguensis & 33 & 1 & s & - & a & + & + & 2 & 2 & nd & 79 & 154 & 6,4 \\
\hline Prosopis affinis & 34 & 2 & s & - & a & + & + & 2 & 2 & nd & 121 & 159 & 6,4 \\
\hline Prosopis nigra & 34 & 2 & $\mathrm{~s}$ & - & a & + & + & 2 & 2 & nd & 11 & 176 & 6,7 \\
\hline Senna corymbosa & 35 & 1,2 & $\mathrm{~s}$ & - & a & + & + & 2 & 2 & nd & 77 & 211 & 5,9 \\
\hline \multicolumn{14}{|l|}{ Flacourtiaceae } \\
\hline Azara uruguayensis & 36 & nd & $\mathrm{m}$ & - & a & - & + & 1 & 2 & nd & 30 & 570 & 7,2 \\
\hline Xylosma tweediana & 37 & 2 & s & + & a & - & + & 1 & 2 & 290 & 41 & 795 & 8,1 \\
\hline \multicolumn{14}{|l|}{ Meliaceae } \\
\hline Guarea macrophylla & 38 & 2 & s & - & a & - & - & 1 & 2 & 13 & 120 & 594 & 3,0 \\
\hline Trichilia claussenii & 39 & 2 & $\mathrm{~s}$ & - & a & - & + & 1 & 2 & 45 & 43 & 410 & 3,1 \\
\hline
\end{tabular}


Continuação da Tabela 1...

\begin{tabular}{|c|c|c|c|c|c|c|c|c|c|c|c|c|c|}
\hline Família / Espécie & RB & H & $\mathbf{P P}$ & EE & API & OPI & $\mathbf{A C}$ & $\mathbf{P}$ & $\mathbf{A P}$ & Fr & D & CEV & DPI \\
\hline \multicolumn{14}{|l|}{ Myrtaceae } \\
\hline Acca sellowiana & 40 & 2 & $\mathrm{~s}$ & - & a & + & + & 1 & 1 & 266 & 33 & 444 & 5,1 \\
\hline Blepharocalyx salicifolius & 41 & 2 & $\mathrm{~s}$ & - & nd & nd & + & 1 & 1 & 70 & 50 & 444 & nd \\
\hline Calyptranthes concinna & 42 & 1,2 & $\mathrm{~s}$ & - & nd & nd & + & 1 & 1 & 11 & 58 & 510 & nd \\
\hline Eugenia involucrata & 43 & 2 & $\mathrm{~s}$ & + & nd & nd & + & 1 & 1 & 21 & 58 & 582 & nd \\
\hline Eugenia mansoi & 44 & 2 & $\mathrm{~s}$ & - & a & - & + & 1 & 2 & 64 & 30 & 592 & 4,4 \\
\hline Eugenia uniflora & 45 & 2 & $\mathbf{s}$ & - & a & - & + & 1 & 1 & 100 & 38 & 524 & 5,0 \\
\hline Myrceugenia euosma & 46 & 1,2 & $\mathrm{~m}$ & + & $\mathrm{a} / \mathrm{o}$ & + & + & 1 & 2 & 266 & 29 & 541 & 9,6 \\
\hline Myrceugenia glaucescens & 47 & 1,2 & $\mathrm{~m}$ & + & $\mathrm{a} / \mathrm{o}$ & + & + & 1 & 1 & 116 & 39 & 596 & 7,5 \\
\hline Myrceugenia myrtoides & 48 & 1 & $\mathrm{~s}$ & + & a & + & + & 1 & 1 & 140 & 43 & 594 & 3,7 \\
\hline Myrcia bombycina & 49 & 1,2 & $\mathrm{~s}$ & + & a & + & + & 1 & 1 & 34 & 58 & 461 & 7,5 \\
\hline Myrcianthes gigantea & 50 & 2 & $\mathrm{~s}$ & - & nd & nd & + & 1 & 1 & 161 & 27 & 542 & nd \\
\hline Myrcianthes pungens & 51 & 2 & $\mathrm{~s}$ & - & a & - & + & 1 & 1 & 136 & 37 & 505 & 4,9 \\
\hline Myrciaria tenella & 52 & 1,2 & $\mathbf{s}$ & - & a & + & + & 2 & 1 & 177 & 29 & 469 & 3,1 \\
\hline Myrrhinium atropurpureum & 53 & 2 & $\mathbf{s}$ & + & nd & nd & + & 1 & 1 & 54 & 42 & 503 & nd \\
\hline Plinia rivularis & 54 & 2 & $\mathrm{~s}$ & - & $\mathrm{a}$ & nd & + & 1 & 2 & 54 & 41 & 545 & 5,0 \\
\hline \multicolumn{14}{|l|}{ Phyllanthaceae } \\
\hline Phyllanthus sellowianus & 12 & 1 & $\mathbf{s}$ & - & a & - & + & 2 & 2 & 179 & 29 & 433 & 4,3 \\
\hline \multicolumn{14}{|l|}{ Polygonaceae } \\
\hline Coccoloba cordata & 55 & 2 & $\mathbf{s}$ & - & a & + & + & 1 & 2 & 50 & 37 & 290 & 3,3 \\
\hline \multicolumn{14}{|l|}{ Quillajaceae } \\
\hline Quillaja brasiliensis & 56 & 2 & $\mathrm{~s}$ & + & a & - & + & 1 & 1 & 30 & 60 & 374 & 5,7 \\
\hline \multicolumn{14}{|l|}{ Rhamnaceae } \\
\hline Colletia paradoxa & 57 & 1 & $\mathrm{~s}$ & + & a & - & + & 1 & 3 & 335 & 25 & 264 & 6,0 \\
\hline Discaria americana & 58 & 1 & $\mathbf{s}$ & + & a & - & + & 1 & 3 & 485 & 31 & 273 & 6,5 \\
\hline Gouania ulmifolia & 59 & 3 & $\mathrm{~s}$ & - & a & - & + & 3 & 2 & 62 & 161 & 187 & 9,3 \\
\hline Rhamnus sphaerosperma & 60 & 2 & $\mathbf{s}$ & + & a & - & + & 2 & 2 & 109 & 46 & 389 & 7,9 \\
\hline Scutia buxifolia & 61 & 2 & $\mathrm{~s}$ & + & a & - & + & 1 & 2 & 82 & 50 & 298 & 3,6 \\
\hline \multicolumn{14}{|l|}{ Rubiaceae } \\
\hline Chomelia obtusa & 62 & 1 & $\mathrm{~s}$ & - & a & + & + & 1 & 2 & 241 & 36 & 716 & 3,6 \\
\hline Guettarda uruguensis & 63 & 1,2 & $\mathbf{s}$ & - & a & + & + & 1 & 2 & 98 & 47 & 638 & 5,0 \\
\hline Machaonia spinosa & 64 & 1 & $\mathrm{~s}$ & - & a & + & + & 2 & 2 & 91 & 47 & 259 & 3,6 \\
\hline Randia ferox & 65 & 1 & $\mathrm{~s}$ & - & a & + & + & 1 & 1 & 80 & 46 & 720 & 4,4 \\
\hline \multicolumn{14}{|l|}{ Rutaceae } \\
\hline Helietta apiculata & 66 & 2 & $\mathbf{s}$ & - & a & - & + & 1 & 2 & 43 & 52 & 299 & 3,7 \\
\hline Pilocarpus pennatifolius & 67 & 1,2 & $\mathbf{s}$ & - & a & - & + & 1 & 2 & 104 & 41 & 417 & 2,7 \\
\hline \multicolumn{14}{|l|}{ Salicaceae } \\
\hline Salix humboldtiana & 68 & 2 & $\mathrm{~s}$ & - & a & - & + & 2 & 2 & 94 & 64 & 386 & 6,0 \\
\hline \multicolumn{14}{|l|}{ Santalaceae } \\
\hline $\begin{array}{l}\text { Acanthosyris spinescens } \\
\text { Sapotaceae }\end{array}$ & 69 & 2 & $\mathrm{~s}$ & - & a & - & + & 1 & 2 & nd & 64 & 243 & 9,3 \\
\hline Pouteria salicifolia & 70 & 2 & $\mathrm{~s}$ & - & a & - & + & 1 & 2 & 57 & 54 & 359 & 4,5 \\
\hline \multicolumn{14}{|l|}{ Solanaceae } \\
\hline Brunfelsia australis & 71 & 1,2 & $\mathrm{~s}$ & - & a & - & + & 1 & 1 & 105 & 35 & 512 & 4,7 \\
\hline \multicolumn{14}{|l|}{ Ulmaceae } \\
\hline Celtis pallida & 72 & 1 & $\mathrm{~s}$ & - & a & - & + & 1 & 2 & 18 & 60 & 167 & 7,2 \\
\hline \multicolumn{14}{|l|}{ Verbenaceae } \\
\hline Cytharexylum solanaceum & 73 & 1,2 & $\mathrm{~m}$ & + & a & - & + & 3 & 2 & 14 & 83 & 264 & 4,0 \\
\hline
\end{tabular}


RB = referência bibliográfica: 1, Marchiori (1985g); 2, Marchiori \& Oliveira (2007); 3, Marchiori et al. (2007); 4, Oliveira et al. (2005b); 5, Oliveira et al. (2005a); 6, Oliveira \& Marchiori (2005); 7, Marchiori (2005b); 8, Marchiori (2009); 9, Marchiori (1986e); 10, Marchiori (1983b); 11, Marchiori \& Denardi (2005a); 12, Denardi (2007); 13, Marchiori (1996a); 14, Marchiori (1992); 15, Marchiori (1993b); 16, Marchiori (1991); 17, Marchiori (1982a); 18, Marchiori (1994); 19, Marchiori (1995a); 20, Marchiori (1986c); 21, Marchiori (1985b); 22, Marchiori (1985i); 23, Marchiori (1996c); 24, Marchiori (1993a); 25, Marchiori (1985e); 26, Marchiori (1982b); 27, Carnieletto \& Marchiori (1993); 28, Marchiori (1996d); 29, Marchiori \& Muñiz (1997b); 30, Marchiori (1995b); 31, Maccari \& Marchiori (1994); 32, Marchiori 7 Muñiz (1997a); 33, Marchiori (1996b); 34, Marchiori (1985f); 35, Marchiori (1983c); 36, Denardi et al. (2005b); 37, Marchiori \& Pozzera (1997); 38, Marchiori (1985d); 39, Marchiori (1985c); 40, Marchiori (1984a); 41, Denardi \& Marchiori (2006); 42, Marchiori \& Brum (1997); 43, Marchiori (1984b); 44, Marchiori \& Santos (2009a); 45, Santos \& Marchiori (2009c); 46, Santos \& Marchiori (2009b); 47, Marchiori \& Muñiz (1988); 48, Marchiori (1987b); 49, Marchiori \& Santos (2009); 50, Marchiori et al. (2008); 51, Santos \& Marchiori (2009d); 52, Marchiori \& Muñiz (1987b); 53, Marchiori (1984d); 54 Denardi et al. (2005a); 55, Marchiori \& Muñiz (1987a); 56, Marchiori (1984c); 57, Marchiori (1983a); 58, Santos et al. (2007a); 59, Santos et al. (2008a); 60, Santos et al. (2007b); 61, Santos et al, 2008b); 62, Marchiori (1986a); 63, Marchiori (1986d); 64, Marchiori \& Muñiz (1987c); 65, Marchiori (1987a); 66, Marchiori (1985a); 67, Marchiori (1986b); 68, Denardi et al. (2007); 69, Marchiori (1985h); 70, Marchiori (1986f); 71, Siegloch et al. (2010); 72, Marchiori \& Freitas (1993); 73, Gomes et al. (2008).

$\mathrm{H}$ = hábito: 1, arbusto; 2, arvoreta/árvore; 3 , liana. $\mathrm{PP}=$ placa de perfuração: $\mathrm{s}$, simples; $\mathrm{m}$, múltipla. $\mathrm{EE}=$ espessamentos espiralados: +, presente; -, ausente. API = arranjo pontoações intervasculares: a, alterno; 0 , oposto. OPI = ornamentações em pontoações intervasculares: +, presente; -, ausente. $\mathrm{AC}=$ anel de crescimento: + , presente; -, ausente. $\mathrm{P}=$ porosidade: 1, difusa; 2 , semi-difusa; 3 , em anel. $\mathrm{AP}=$ arranjo/agrupamento de poros: 1 , exclusivamente solitários/sem padrão definido de organização; 2 , múltiplos radiais/racemiformes; 3 , padrão diagonal/dendrítico. $\mathrm{Fr}=$ freqüência média de poros $\left(\right.$ poros $\left./ \mathrm{mm}^{2}\right)$. $\mathrm{D}=$ diâmetro médio de poros $(\mu \mathrm{m})$. CEV $=$ comprimento médio de elementos vasculares $(\mu \mathrm{m})$. DPI = diâmetro médio pontoações intervasculares $(\mu \mathrm{m})$. $\mathrm{nd}=$ não disponível.

poros em múltiplos radiais/racemiformes, $22 \%$ não exibem padrão definido de organização e, em apenas $12 \%$ ocorre o padrão diagonal ou dendrítico. No material analisado, os múltiplos radiais e racemiformes encontram-se geralmente em combinação com poros solitários.

Cabe salientar, de início, que o arranjo de poros está fortemente relacionado ao grupo taxonômico. Em Asteraceae, por exemplo, os padrões dendrítico ou em múltiplos radiais e racemiformes são largamente predominantes; nas Leguminosae, a presença de poros solitários e em múltiplos radiais/racemiformes verificase na maioria dos gêneros; em Myrtaceae, por sua vez, é nítida a tendência de formar poros solitários (Record \& Hess, 1949; Metcalfe \& Chalk, 1972). No presente estudo, o predomínio de poros em múltiplos radiais/racemiformes pode ser atribuído, pelo menos em parte, ao grande número de Leguminosae incluídas na pesquisa. Mesmo assim, cabe lembrar que das 24 famílias relacionadas, 19 possuem representantes com poros em múltiplos radiais/ racemiformes, comprovando ser este o padrão predominante nas Dicotiledôneas nativas. Os resultados de Barros et al. $(2001 ; 2003)$ e de Mattos et al. (2006), confirmam esta tendência. Tal predomínio, todavia, não deve ser interpretado como mera coincidência, mas como decorrência de um processo seletivo na constituição de um dado bioma. Pode-se notar, inclusive, uma estreita relação com o hábito de crescimento: todas as espécies com poros em arranjo diagonal/dendrítico, no presente estudo, são de hábito arbustivo, em consonância com tendência previamente reconhecida por Calquist (1966).

\section{Frequência de poros}

A maioria das espécies investigadas (63\%) apresenta mais de 40 poros $/ \mathrm{mm}^{2}$. Reduzindo-se o limite inferior para 20 poros $/ \mathrm{mm}^{2}$, o percentual sobe para $80 \%$ das espécies, superando os valores comumente referidos para florestas tropicais úmidas (Carlquist, 1975; 2001; Pérez, 1989; Wheeler \& Baas, 1991; Wheeler et al, 2007). No tocante à flora brasileira, Barros et al. (2006) encontraram menos de 20 poros $/ \mathrm{mm}^{2}$ em $92 \%$ das espécies, em remanescente florestal do Rio de Janeiro. Para o Pantanal Mato- 
Grossense, Mattos et al. (2003) observaram uma frequiência de até 20 poros $/ \mathrm{mm}^{2}$ em mais de $70 \%$ das espécies. Resultados semelhantes foram obtidos por Woodcock et al. (2000), em diferentes tipologias florestais da Amazônia peruana, bem como por León (2005), em estudo de um bosque seco tropical na Venezuela.

No presente estudo, a elevada frequência de poros pode ser explicada, ao menos em parte, pela maior latitude do Estado. De acordo com Noshiro \& Baas (2000), o comportamento dos vasos, em relação à latitude, assemelha-se ao observado na direção do gradiente mésicoxérico, onde verifica-se aumento na frequiência de poros, tendência confirmada nos trabalhos de Baas (1973), Baas et al. (1983), Lindorf (1994), Wheeler \& Baas (1991) e Wheeler et al. (2007).

Cabe salientar, ainda, a elevada freqüência de poros (> 200 poros $/ \mathrm{mm}^{2}$ ) em arbustos com vasos em padrão dendrítico, aspecto que pode ser atribuído tanto ao hábito quanto à especialização xeromórfica (Carlquist, 1975; Baas \& Schweingruber, 1987).

\section{Elementos vasculares}

Placas de perfuração simples foram encontradas em aproximadamente $94 \%$ das espécies, percentual semelhante ao referido por Alves \& Angyalossy-Alfonso (2000), Mattos et al. (2003) e Barros et al. (2006), para outras regiões do Brasil. Esta percentagem confirma a conhecida prevalência do caráter em Dicotiledôneas, sobretudo em Florestas Tropicais de Terras Baixas (Carlquist \& Hoekman, 1985; Baas \& Schweingruber, 1987; Wheeler \& Baas, 1991).

A presença de placas múltiplas, caráter minoritário em Dicotoledôneas, vincula-se a determinados grupos taxonômicos, notadamente a certos gêneros e espécies (Metcalfe \& Chalk, 1983). Entre as Mirtáceas nativas, por exemplo, apenas Myrceugenia inclui o referido caráter. Freqüências elevadas de placas múltiplas são comuns em floras de montanhas tropicais e de regiões temperadas, sugerindo uma estreita relação do caráter com altitudes e/ou latitudes elevadas (Carlquist \& Hoekman, 1985; Baas \& Schweingruber, 1987; Wheeler et al., 2007). A ocorrência restrita de placas múltiplas em Florestas Tropicais Úmidas pode ser compreendida, em termos fisiológicos, por sua menor eficiência no transporte de água, aspecto desvantajoso sob taxas condutivas elevadas (Carlquist, 1975; Wheeler \& Baas, 1991). Apesar da posição austral do Rio Grande do Sul no contexto brasileiro, o percentual de espécies com placas múltiplas (cerca de 6\%) assemelhase ao referido por Alves \& Angyalossy-Alfonso (2000) para o norte do País; este resultado, todavia, pode dever-se à questões de amostragem, uma vez que a ocorrência do caráter concentra-se em determinados grupos taxonômicos.

A presença de espessamentos espiralados é mais frequiente em regiões temperadas (Carlquist, 1975; Baas et al., 1983; Baas \& Schweingruber, 1987; Wheeler et al., 2007) e em ambientes secos (Webber, 1936; Carlquist, 1966; Carlquist \& Hoekman, 1985); uma relação com altitude tem sido igualmente postulada, por alguns autores (Graaf \& Baas, 1974; Metcalfe $\&$ Chalk, 1983). No presente estudo, cerca de $30 \%$ das espécies investigadas apresentam espessamentos espiralados na parede dos vasos, em consonância com a distribuição latitudinal do caráter. Esta tendência é confirmada em estudo de Alves \& Angyalossy-Alfonso (2000), uma vez que as autoras também encontraram um percentual maior de espécies com espessamentos espiralados na região sul do Brasil; comparado aos valores referidos, todavia, o percentual presentemente observado mostra-se significativamente maior, fato que pode ser atribuído ao elevado número de espécies de grupos taxonômicos com estrutura especializada, caso dos gêneros Heterothalamus e Baccharis (Asteraceae).

Com relação ao comprimento, observou-se que $54 \%$ das espécies analisadas possuem 
elementos vasculares curtos $(<350 \mu \mathrm{m})$; as demais têm comprimento médio (350-800 $\mu \mathrm{m})$. Para remanescentes florestais no Rio de Janeiro, Barros et al. $(2003 ; 2006)$ referem uma frequiência maior de espécies com elementos vasculares de 350-800 $\mu \mathrm{m}$ de comprimento, à semelhança do observado por Woodcock et al. (2000) na Amazônia peruana, e por León (2005), em um bosque seco da Venezuela. Elementos vasculares curtos são mais freqüentes em ambientes secos (Carlquist, 1975; Baas \& Carlquist, 1985; Carlquist \& Hoekman, 1985; Lindorf, 1994) ou em latitudes elevadas (Baas, 1973; Wilkins \& Papassotiriou, 1989; Noshiro $\&$ Baas, 2000; Wheeler et al., 2007), do que em ambientes mésicos, de regiões tropicais (Baas et al., 1983; Barajas-Morales, 1985; León, 2005). A predominância de elementos vasculares curtos em espécies sul-rio-grandenses, comparado a outras floras brasileiras, corrobora a hipótese da redução do caráter com o aumento da latitude, sobretudo em clima ombrófilo, como o do Rio Grande do Sul. Contrariamente a esta tendência, a maioria das espécies relacionadas por Mattos et al. (2003) apresenta elementos vasculares curtos. Cabe salientar, todavia, que o grupo taxonômico pode ter grande influência no comprimento de elementos vasculares. Em Leguminosae e Compositae (Asteraceae), por exemplo, predominam elementos vasculares curtos (Metcalfe \& Chalk, 1972), ao contrário de Myrtaceae, família com nítida predominância de elementos vasculares médios (Vliet \& Baas, 1984). Deste modo, o predomínio de elementos vasculares curtos, observado no presente estudo, pode ser atribuído, pelo menos em parte, ao grande número de Fabaceae e Asteraceae investigadas; o mesmo pode-se dizer dos resultados de Mattos et al. (2003).

A maioria das espécies analisadas (cerca de $51 \%)$ apresenta poros muito pequenos $(<50$ $\mu \mathrm{m})$, seguindo-se os intervalos de $50-100 \mu \mathrm{m}$ $(36 \%)$ e das madeiras com vasos maiores do que $100 \mu \mathrm{m}(13 \%)$. Para o Pantanal Mato-Grossense, Mattos et al. (2003) referem diâmetro médio de
50 a $100 \mu \mathrm{m}$ para a maioria das espécies (cerca de $62 \%$ ), sendo que em apenas $15 \%$ das madeiras os poros são menores do que $50 \mu \mathrm{m}$. Em remanescente de Floresta Atlântica (Rio de Janeiro), Barros et al. (2006) constataram o predomínio de espécies com vasos maiores do que $100 \mu \mathrm{m}(69 \%)$ de diâmetro, resultado semelhante ao de Cury (2002), para espécies arbóreas do Cerrado e Mata Atlântica de São Paulo (59\%). Poros com mais de $100 \mu \mathrm{m}$ de diâmetro são típicos de espécies mésicas (Carlquist, 1975; Barajas-Morales, 1985; Woodcock et al., 2000; Wheeler et al., 2007), diferentemente de representantes xéricos, de terras altas tropicais, de climas temperados e de latitudes elevadas, para os quais verificam-se valores geralmente inferiores a $50 \mu \mathrm{m}$ (Baas, 1973; Barajas-Morales, 1985; Wheeler \& Baas, 1991; Lindorf, 1994; Carlquist, 2001).

Considerando-se a classe de diâmetro predominante, os valores presentemente observados são significativamente menores do que o mencionado para as floras brasileiras acima referidas, de caráter mésico. Tal comportamento pode ser explicado, sob o ponto de vista fisiológico, pelas menores taxas condutivas decorrentes da posição latitudinal, posto que o Rio Grande do Sul é o estado mais austral do Brasil. Contrariamente, em direção aos trópicos, onde as taxas condutivas são mais elevadas, os poros tendem a aumentar em diâmetro, com vistas a garantir maior eficiência na condução (Zimmermann, 1982).

A grande maioria das espécies investigadas (cerca de 96\%) apresenta pontoações intervasculares alternas, uma tendência geral em Dicotiledôneas (IAWA, 1989; Wheeler et al., 2007), comprovada, inclusive, em madeiras brasileiras (Mattos et al., 2003; Barros et al., 2006). O contingente com pontoações ornamentadas é relativamente alto no presente estudo (cerca de 49\%), comparado ao referido por Mattos et al. (2003), Barros et al. (2006) e Wheeler et al. (2007) $(15,31$ e $30 \%$, respectivamente). Vinculada a determinados grupos 
taxonômicos (Jansen et al., 2001), a alta percentagem de espécies com ornamentações, comprovada no presente estudo, pode ser atribuída aos numerosos representantes de Leguminosae e Myrtaceae providos do referido caráter anatômico. Com relação ao diâmetro, a maioria das espécies apresenta pontoações intervasculares pequenas (56\%), seguindo-se pontoções médias (29\%) e diminutas (15\%), à semelhança do observado na flora mundial (Wheeler et al., 2007). Como no caso das ornamentações, o diâmetro de pontoações intervasculares também é caráter conservativo para espécies, gêneros e famílias botânicas, independentemente do local de ocorrência (Marcati et al., 2001; León, 2005). A variabilidade observada, desse modo, pode ser atribuída à diversidade e predomínio de alguns grupos taxonômicos no material em estudo.

\section{CONCLUSÕES}

Anéis de crescimento distintos, placas de perfuração simples e pontoações intervasculares alternas são amplamente dominantes em madeiras sul-rio-grandenses, constando em mais de $90 \%$ das espécies investigadas. São igualmente predominantes: porosidade difusa; poros em múltiplos, em arranjo radial/ racemiforme; poros muito pequenos $(<50 \mu \mathrm{m})$, com frequência superior a $20 / \mathrm{mm}^{2}$; elementos vasculares curtos $(<350 \mu \mathrm{m})$; e pontoações intervasculares pequenas $(4-7 \mu \mathrm{m})$, não ornamentadas. $\mathrm{O}$ arranjo de poros, a presença de ornamentações em pontoações intervasculares, bem como de espessamentos espiralados em vasos podem ser atribuídos, pelo menos em parte, ao elevado número de espécies estudadas, pertencentes a determinados grupos taxonômicos. Comparado a floras de outras regiões do Brasil, as diferenças observadas no tocante à porosidade, frequiência, diâmetro $\mathrm{e}$ comprimento de elementos vasculares podem ser explicadas, em princípio, pelo posicionamento austral do Estado no país.

\section{BIBLIOGRAFIA CONSULTADA}

ALVES, E.S.; ANGYALOSSY-ALFONSO, V. Ecological trends in the wood anatomy of some brazilian species. 1. Growth rings and vessels. IAWA Journal, v. 21, p. 3-30, 2000.

ALVES, E.S.; ANGYALOSSY-ALFONSO, V. Ecological trends in the wood anatomy of some brazilian species. 2. Axial parenchyma, rays and fibers. IAWA Journal, v. 23, n. 4, p. 391-418, 2002.

BAAS, P. The wood anatomical range in Ilex (Aquifoliaceae) and its ecological and phylogenetic significance. Blumea, v. 21, n. 2, p. 193-258, 1973.

BAAS, P.; CARLQUIST, S. A comparison of the ecological wood anatomy of the floras of Southern California and Israel. IAWA Bulletin, v. 6 , p. $349-353,1985$.

BAAS, P.; SCHWEINGRUBER, F.H. Ecological trends in wood anatomy of trees, shrubs and climbers from Europe. IAWA Bulletin, v. 8, p. 245-274, 1987.

BAAS, P.; WERKER, E.; FAHN, A. Some ecological trends in vessel characters. IAWA Bulletin, v. 4, p. 141-160, 1983.

BARAJAS-MORALES, J. Wood structural differences between trees of two tropical forests in Mexico. IAWA Bulletin, v. 6, n. 4, p. 355-364, 1985.

BARROS, C.F.; CALLADO, C.H.; CUNHA, M.; MARCON, M.L.F.; TAMAIO, N.; MARQUETE, O.; COSTA, C.G. Madeiras da Mata Atlântica. Anatomia do lenho de espécies ocorrentes nos remanescentes florestais do estado do Rio de Janeiro, Brasil. Rio de Janeiro: Instituto de Pesquisas Jardim Botânico do Rio de Janeiro, 2003. 86 p.

BARROS, C.F.; CALLADO, C.H.; MARCON, M.L.; COSTA, C.G.; CUNHA, M.; LIMA, H.R.P.; MARQUETE, O. Madeiras da Mata Atlântica. Anatomia do lenho de espécies ocorrentes nos remanescentes florestais do estado do Rio de Janeiro, Brasil. Rio de Janeiro: Instituto de Pesquisas Jardim Botânico do Rio de Janeiro, 2001. 94 p.

BARROS, C.F.; MARCON-FERREIRA, M.L.; CALLADO, C.H.; LIMA, H.R.P.; CUNHA, M.; MARQUETE, O.; COSTA, C.G. Tendências ecológicas na anatomia da madeira de espécies da comunidade arbórea da Reserva Biológica de 
Poço das Antas, Rio de Janeiro, Brasil. Rodriguésia, v. 57, n. 3, p. 443-460, 2006.

BRASIL, Ministério da Agricultura. Departamento Nacional de Pesquisa Agropecuária. Divisão Pedológica. Levantamento de reconhecimento dos solos do estado do Rio Grande do Sul. (Boletim técnico n. 30). Recife, PE, 1973. 431 p.

CALLADO, C.H.; NETO, S.J.S.; SCARANO, F.R.; BARROS, C.F.; COSTA, S.G. Anatomical features of growth rings in flood-prone trees of the Atlantic Rain Forest in Rio de Janeiro, Brazil. IAWA Journal, v. 22, n. 1, p. 29-42, 2001.

CARLQUIST, S. Wood anatomy of Compositae: a summary with comments on factors controlling wood evolution. Aliso, v. 6, n. 2, p.25-44, 1966.

CARLQUIST, S. Ecological strategies of xylem evolution. Berkeley: University of California Press, 1975. $259 \mathrm{p}$.

CARLQUIST, S. Vessel grouping in Dicotyledon woods. Significance and relationship to imperforate tracheary elements. Aliso, v. 10, p. 505-525, 1984.

CARLQUIST, S. Comparative Wood Anatomy. Systematic, ecological and evolutionary aspects of Dicotyledon woods. Berlin: Springer-Verlag, 2001. $446 \mathrm{p}$.

CARLQUIST, S.; HOEKMAN, D.A. Ecological wood anatomy of the woody southern californian flora. IAWA Bulletin, v. 6, n. 4, p. 319-347, 1985.

CARNIELETTO, C.; MARCHIORI, J.N.C. Anatomia da madeira de Mimosa eriocarpa Benth. Ciência Florestal, Santa Maria, v. 3, n. 1, p. 107120, 1993.

CURY, G. Descrição da estrutura anatômica do lenho e sua aplicação na identificação de espécies arbóreas do cerrado e da mata atlântica do estado de São Paulo. Piracicaba: ESALQ, 2002. 125 f. Dissertação (Mestrado) - Universidade de São Paulo: Escola Superior de Agricultura Luiz de Queiroz.

DENARDI, L. Anatomia e flexibilidade do caule de quatro espécies lenhosas, para o manejo biotécnico de cursos de água. Santa Maria: UFSM, 2007. 113 p. Tese (Doutorado em Manejo Florestal) - Universidade Federal de Santa Maria.

DENARDI, L. MARCHIORI, J.N.C. Anatomia do lenho da murta, Blepharocalyx salicifolius (H.B.K.) Berg. Ciência Florestal, Santa Maria, v. 15 , n. 3, p. 267-274, 2006.

DENARDI, L.; MARCHIORI, J.N.C.; FERREIRA, M.R. Anatomia da madeira de Plinia rivularis (Camb.) Rotman. Balduinia, Santa Maria, n. 3, p. 21-25, 2005a.

DENARDI, L.; MARCHIORI, J.N.C.; FERREIRA, M.R. Anatomia da madeira de Azara uruguayensis (Speg.) Sleum. Balduinia, Santa Maria, n. 5, p. 17-22, 2005 b.

DENARDI, L.; MARCHIORI, J.N.C.; SANTOS, S.R. dos. Anatomia da madeira de Salix humboldtiana Willd. (Salicaceae). Balduinia, Santa Maria, n. 11, p. 27-31, 2007.

GOMES, A.V.; MARCHIORI, J.N.C.; TEIXEIRA, L.L.; SCHAITZA, E.G.; STOFELLA, D.R.E. Anatomia da madeira de Citharexylum solanaceum Cham. (Verbenaceae). Balduinia, Santa Maria, n. 12, p. 13-25, 2008.

GOMEZ, A.V; MUÑIZ, G.I.B. Wood structure and ultrastructure of Prosopis caldenia, P. chilensis and $P$. juliflora and influence of ecological factors. In: Prosopis International Conference, 2, 1986. Recife, Pernambuco.

GRAAF, N.A.; BAAS, P. Wood anatomical variation in relation to latitude and altitude. Blumea, v.22, p. 101-121, 1974.

HERENDEEN, P.S., WHEELER, E.A., BAAS, P. Angiosperm wood evolution and the potential contribution of paleontological data. The Botanical Review, v. 65, n. 3, p. 278-300, 1999.

IAWA COMMITTEE. IAWA list of microscopic features for hardwood identificacion. IAWA Bulletin, v. 10, n. 3, p. 218-359, 1989.

JANSEN, S.; BAAS, P.; SMETS, E. Vestured pits. Their occurrence and systematic importance in Eudicots. Taxon, v. 50, n. 1, p. 135-167, 2001.

LEÓN, W. Anatomía ecológica del xilema secundario de un bosque seco tropical de Venezuela. Acta Botánica Venezuelica, v. 28, n. 2, p. 1-22, 2005.

LINDORF, H. Eco-anatomical wood features of species from a very dry tropical forest. IAWA Bulletin, v. 15, p. 361-376, 1994.

LISI, C.S.; TOMAZELLO FILHO, M.; BOTOSSO, P.C.; ROIG, F.A.; MARIA, V.R.B.; FERREIRAFEDELE, L.; VOIGT, A.R.A. Tree-ring formation, radial increment periodicity, and phenology of tree species from a Seasonal Semi-Deciduous Forest in southeast Brazil. IAWA Journal, v. 29, p. 189-207, 2008. 
MACCARI, A.; MARCHIORI, J.N.C. Estudo anatômico do xilema secundário de Mimosa sparsa Benth. Ciência Florestal, Santa Maria, v. 4, p. 145-155, 1994.

MAINIERI, C.; CHIMELO, J.P. Fichas de características das madeiras brasileiras. São Paulo: IPT - Divisão de Madeiras,1989. 418 p.

MALUF, J.R.T. Nova classificação climática do Estado do Rio Grande do Sul. Revista Brasileira de Agrometeorologia, Santa Maria, v. 8, n. 1, p. 141-150, 2000.

MARCATI, C.R.; ANGYALOSSY-ALFONSO, V.; BENETATI, L. Anatomia comparada do lenho de Copaifera langsdorffii Desf. (Leguminosae - Caesalpinoideae) de floresta e cerradão. $R e$ vista Brasileira de Botânica, v. 24, n. 3, p. 311320, 2001.

MARCATI, C.R.; OLIVEIRA, J.S.; MACHADO, S.R. Growth rings in Cerrado woody species. Occurrence and anatomical markers. Biota Neotropica, v. 6, n. 3, p. 1-31, 2006.

MARCHIORI, J.N.C. Estudo anatômico do xilema secundário e da casca de Acacia recurva Benth. (Leguminosae Mimosoideae). Ciência e Natura, Santa Maria, v. 4, p. 95-105, 1982a.

MARCHIORI, J.N.C. A estrutura do xilema secundário de Mimosa daleoides Benth. (Leguminosae Mimosoideae). Ciência e Natura, Santa Maria, v. 4, p. 107-113, 1982 b.

MARCHIORI, J.N.C. Anatomia da madeira de Colletia paradoxa (Spreng.) Escalante. Ciência e Natura, Santa Maria, v. 5, p. 161-170, 1983a.

MARCHIORI, J.N.C. Anatomia da madeira de Maba inconstans (Jacq.) Gris. (Ebenaceae). Ciência e Natura, Santa Maria, v. 5, p. 153-160, 1983b.

MARCHIORI, J.N.C. Estudo anatômico do xilema secundário de Cassia corymbosa Lam. (Leguminosae Caesalpinioideae). Ciência e Natura, Santa Maria, v. 5, p. 179-186, 1983c.

MARCHIORI, J.N.C. Anatomia descritiva do lenho de Feijoa sellowiana Berg. Ciência e Natura, Santa Maria, v. 6, p. 117-125, 1984a.

MARCHIORI, J.N.C. Anatomia da madeira de Eugenia involucrata DC. (Myrtaceae). Ciência e Natura, Santa Maria, v. 6, p. 127-136, 1984b.

MARCHIORI, J.N.C. Anatomia descritiva da madeira de pau-de-sabão, Quillaja brasiliensis (St. Hil. et Tul.) Mart., Rosaceae Spiraeoideae. Rev. Centro de Ciências Rurais, Santa Maria, v. 14, n. 1, p. 33-41, 1984c.
MARCHIORI, J.N.C. Anatomia descritiva da madeira de murtilho, Myrrhinium loranthoides (Hook. et Arn.) Burret, Myrtaceae. Rev. Centro de Ciências Rurais, Santa Maria, v. 14, n. 1, p. 43-50, 1984d.

MARCHIORI, J.N.C. Anatomia descritiva da madeira de canela-de-veado, Helietta longifoliata Britton, Rutaceae. Rev. Centro de Ciências Rurais, Santa Maria, v. 15, n. 4, p. 335-344, 1985a.

MARCHIORI, J.N.C. Estudo anatômico da madeira de Lonchocarpus leucanthus Burk. (Leguminosae Faboideae). Rev. Centro de Ciências Rurais, Santa Maria, v. 15, n. 4, p. 345-354, 1985 b.

MARCHIORI, J.N.C. Anatomia da madeira de catiguá-vermelho, Trichilia hieronymi Griseb. (Meliaceae). Rev. Centro de Ciências Rurais, Santa Maria, v. 15, n. 4, p. 355-365, 1985 c.

MARCHIORI, J.N.C. Anatomia descritiva da madeira de Guarea lessoniana A. Juss. Ciência e Natura, Santa Maria, v. 7, p. 63-71, 1985d.

MARCHIORI, J.N.C. Anatomia da madeira de $\mathrm{Mi}$ mosa cruenta Benth. (Leguminosae Mimosoideae). Ciência e Natura, Santa Maria, v. 7 , p. 73-81, 1985e.

MARCHIORI, J.N.C. Anatomia descritiva das madeiras do gênero Prosopis L., nativas no estado do Rio Grande do Sul. Ciência e Natura, Santa Maria, v. 7, p. 83-97, $1985 f$.

MARCHIORI, J.N.C. Anatomia descritiva da madeira de quebracho-branco (Aspidosperma quebracho-blanco Schltdl.). Ciência e Natura, Santa Maria, v. 7, p. 99-106, 1985g.

MARCHIORI, J.N.C. Estudo anatômico da madeira de Acanthosyris spinescens (Mart. et Eichl.) Gris. (Santalaceae). Ciência e Natura, Santa Maria, v. 7, p. 107-118, 1985h.

MARCHIORI, J.N.C. Estudo anatômico da madeira de grapiapunha-do-banhado, Lonchocarpus nitidus (Vogel) Benth. Ciência e Natura, Santa Maria, v. 7, p. 119-127, 1985 i.

MARCHIORI, J.N.C. Estudo anatômico da madeira de Chomelia obtusa Cham. et Schltdl. Ciência e Natura, Santa Maria, v. 8, p. 87-94, 1986a.

MARCHIORI, J.N.C. Estudo anatômico da madeira de Pilocarpus pennatifolius Lam. (Rutaceae). Ciência e Natura, Santa Maria, v. 8, p. 95-103, $1986 \mathrm{~b}$.

MARCHIORI, J.N.C. Anatomia do xilema secundário de Indigofera suffruticosa Miller. Ciência e Natura, Santa Maria, v. 8, p. 105-114, 1986c. 
MARCHIORI, J.N.C. Estudo anatômico da madeira de veludinho, Guettarda uruguensis Cham. et Schltdl. Ciência e Natura, Santa Maria, v. 8, p. 115-123, 1986d.

MARCHIORI, J.N.C. Anatomia descritiva da madeira do amarilho, Terminalia australis Camb. (Combretaceae). Rev. Centro de Ciências Rurais, Santa Maria, v. 16, n. 4, p. 329-340, 1986 e.

MARCHIORI, J.N.C. Estudo anatômico da madeira do mata-olho, Pouteria salicifolia (Spreng.) Radlk. Rev. Centro de Ciências Rurais, Santa Maria, v. 16, n. 4, p. 341-352, $1986 f$.

MARCHIORI, J.N.C. Anatomia da madeira do limoeiro-do-mato, Randia armata (Sw.) DC. Rev. Centro de Ciências Rurais, Santa Maria, v. 17, n. 1-2, p. 39-48, 1987a.

MARCHIORI, J.N.C. Anatomia descritiva da madeira de Myrceugenia myrtoides Berg. Ciência e Natura, Santa Maria, v. 9, p. 113-120, 1987 b.

MARCHIORI, J.N.C. Anatomia da madeira de Acacia nitidifolia Speg. (Leguminosae Mimosoideae). Ciência Florestal, Santa Maria, v. 1, n.1, p. 46-63, 1991.

MARCHIORI, J.N.C. Anatomia da madeira e casca do espinilho, Acacia caven (Mol.) Mol. Ciência Florestal, Santa Maria, v. 2, n. 1, p. 27-47, 1992.

MARCHIORI, J.N.C. Anatomia da madeira e casca do maricá, Mimosa bimucronata (DC.) O. Kuntze. Ciência Florestal, Santa Maria, v. 3, n. 1, p. 85-106, 1993a.

MARCHIORI, J.N.C. Estudo anatômico do xilema secundário de Acacia ibirocayensis Marchiori (Leguminosae Mimosoideae). Ciência e Natura, Santa Maria, v. 15, p. 149-159, 1993b

MARCHIORI, J.N.C. Anatomia da madeira e casca de Acacia tucumanensis Gris. Ciência e Natura, Santa Maria, n. 16, p. 85-104, 1994.

MARCHIORI, J.N.C. Anatomia da madeira do angico-branco, Albizia austrobrasilica Burk. Ciência e Natura, Santa Maria, v. 17, p. 75-86, 1995a.

MARCHIORI, J.N.C. Anatomia da madeira e casca da bracatinga, Mimosa scabrella Benth. Ciência e Natura, Santa Maria, v. 17, p. 115-132, 1995 b.

MARCHIORI, J.N.C. Anatomia da madeira de Acacia bonariensis Gill. ex Hook. et Arn. Ciência Rural, Santa Maria, v. 26, n. 2, p. 209-216, 1996a.

MARCHIORI, J.N.C. Anatomia do xilema secundário de Mimosa uruguensis Hook. et Arn. Ciência e Natura, Santa Maria, v. 18, p. 103-115, 1996b.

MARCHIORI, J.N.C. Anatomia do xilema secundário de Mimosa berroi Burk. Ciência e Natura, Santa Maria, v. 18, p. 117-129, 1996 c.

MARCHIORI, J.N.C. Anatomia do xilema secundário de Mimosa incana (Spreng.) Benth. Ciência Florestal, Santa Maria, v. 6, n. 1, p. 53-63, 1996d.

MARCHIORI, J.N.C. Fitogeografia do Rio Grande do Sul. Enfoque histórico e sistemas de classificação. Porto Alegre: EST, 2002. 118 p.

MARCHIORI, J.N.C. Estudo anatômico da madeira de Handroanthus pulcherrimus (Sandwith) S. Grose. Balduinia, Santa Maria, n. 19, p. 10-13, 2009.

MARCHIORI, J.N.C.; BRUM, E.T. Anatomia da madeira do guamirim-facho, Calyptranthes concinna DC. Ciência Rural, Santa Maria, v. 27, n. 2, p. 217-222, 1997.

MARCHIORI, J.N.C.; DENARDI, L. Anatomia da madeira de Escallonia megapotamica Sprengel. Balduinia, Santa Maria, n. 2, p. 19-24, 2005a.

MARCHIORI, J.N.C.; DENARDI, L. Anatomia da madeira de Berberis laurina Billb. Balduinia, Santa Maria, n. 4, p. 25-29, 2005 b.

MARCHIORI, J.N.C.; DENARDI, L.; FERREIRA, M.R. Anatomia da madeira de Myrcianthes gigantea (Legr.) Legr. Balduinia, Santa Maria, n. 12, p. 27-31, 2008.

MARCHIORI, J.N.C.; FREITAS, A. de M. Anatomia da madeira de Celtis pallida Torrey (Ulmaceae). Ciência e Natura, Santa Maria, v. 15 , p. 137-147, 1993.

MARCHIORI, J.N.C.; MUÑIZ, G.I.B. de. Estudo anatômico da madeira de Coccoloba cordata Cham. (Polygonaceae). Ciência e Natura, Santa Maria, v. 9, p. 87-95, 1987a.

MARCHIORI, J.N.C.; MUÑIZ, G.I.B. de. Estudo anatômico da madeira de Myrciaria tenella (DC.) Berg. Ciência e Natura, Santa Maria, v. 9 , p. $97-103,1987 \mathrm{~b}$.

MARCHIORI, J.N.C.; MUÑIZ, G.I.B. de. Estudo anatômico da madeira de Machaonia spinosa Cham. et Schltdl. (Rubiaceae). Ciência e Natura, Santa Maria, v. 9, p. 105-112, 1987c. 
MARCHIORI, J.N.C.; MUÑIZ, G.I.B. de. Estudo anatômico da madeira de Myrceugenia glaucescens (Camb.) Legr. et Kaus. Ciência e Natura, Santa Maria, v. 10, p. 105-113, 1988.

MARCHIORI, J.N.C.; MUÑIZ, G.I.B. de. Estudo anatômico do xilema secundário de Mimosa trachycarpa Benth. Ciência Rural, Santa Maria, v. 27, n. 2, p. 223-228, 1997a.

MARCHIORI, J.N.C.; MUÑIZ, G.I.B. de. Anatomia do xilema secundário de Mimosa pilulifera Benth. Ciência Florestal, Santa Maria, v. 6, n. 1, p. 65-75, 1997b.

MARCHIORI, J.N.C.; OLIVEIRA, A.S. de. Anatomia da madeira de três espécies brasileiras de Baccharis L. (Asteraceae-Astereae). Balduinia, Santa Maria, n. 10, p. 1-10, 2007.

MARCHIORI, J.N.C.; OLIVEIRA-DEBLE, A.S. Anatomia da madeira da subtribo Baccharinae Less. Tendências ecológicas de ordem taxonômica e ecológica. Balduinia, Santa Maria, n. 11, p. 9-15, 2007.

MARCHIORI, J.N.C.; OLIVEIRA-DEBLE, A.S. de; DENARDI, L. Anatomia da madeira de duas espécies de Baccharis L. (Asteraceae - Astereae). Balduinia, Santa Maria, n. 11, p. 1-8, 2007.

MARCHIORI, J.N.C.; POZZERA, F. Anatomia da madeira de Xylosma tweedianum Eichler (Flacourtiaceae). Ciência e Natura, Santa Maria, v. 19, p. 159-171, 1997.

MARCHIORI, J.N.C.; SANTOS, S.R. dos. Anatomia do xilema secundário de Eugenia mansoi O. Berg (Myrtaceae). Balduinia, Santa Maria, n. 16, p. 6-12, 2009a.

MARCHIORI, J.N.C.; SANTOS, S.R. dos. Descrição anatômica da madeira de Myrcia bombycina (O. Berg) Niedenzu (Myrtaceae). Balduinia, Santa Maria, n. 17, p. 17-22, 2009.

MATTOS, P.P.; TEIXEIRA, L.L.; SEITZ, R.A.; SALIS, M.S.; BOTOSSO, C.P. Anatomia de madeiras do Pantanal Mato-Grossense. Características microscópicas. Colombo: EMBRAPA Florestas. Corumbá: EMBRAPA Pantanal, 2003, $182 \mathrm{p}$.

METCALFE, C.R.; CHALK, L. Anatomy of the Dicotyledons. Oxford: Clarendon Press, 1972. $1500 \mathrm{p}$.

METCALFE, C.R.; CHALK, L. Anatomy of the Dicotyledons. Wood structure and conclusions of the general introdution. Oxford: Clarendon Press, 1983.
NOSHIRO, S.; BAAS, P. Latitudinal trends in wood anatomy within species and genera. Case study in Cornus S. L. (Cornaceae). American Journal of Botany, v. 87, n. 10, p. 1495-1506, 2000.

OLIVEIRA, A.S. de; MARCHIORI, J.N.C. Anatomia do lenho de Hetherothalamus psiadioides Lessing (Asteraceae - Astereae). Balduinia, Santa Maria, n. 4, p. 20-24, 2005.

OLIVEIRA, A.S. de; DEBLE, L.P.; MARCHIORI, J.N.C. Anatomia da madeira de duas espécies do gênero Heterothalamus Lessing (Asteraceae), nativas no Rio Grande do Sul. Ciência Florestal, Santa Maria, v. 15, n. 1, p. 9-19, 2005a.

OLIVEIRA, A.S. de; MARCHIORI, J.N.C.; DEBLE, L.P. Anatomia do lenho de Heterothalamus wagenitzii (F. Hellw.) Deble, Oliveira \& Marchiori (Astereae - Asteraceae). Balduinia, Santa Maria, n. 2, p. 13-18, 2005 b.

PÉREZ, A. Caracterización ecoanatómica del leño de 40 especies del bosque La Mucuy, Estado de Mérida,Venezuela. Revista Forest. Venez., v. 33, p. 43-51, 1989.

RECORD, S.J.; HESS R.W. Timbers of the New World. New Haven: Yale University Press, 1949. $640 \mathrm{p}$.

REITZ, R.; KLEIN, R.M.; REIS, A. Projeto Madeira do Rio Grande do Sul. Sellowia, Itajaí, n. 3435, p. 1-525, 1983.

SANTOS, S.R. dos; MARCHIORI, J.N.C. Estudos anatômicos em Rhamnaceae. Considerações taxonômicas, ecológicas e evolutivas. Balduinia, n. 16 , p. 13-23, 2009a.

SANTOS, S.R. dos; MARCHIORI, J.N.C. Anatomia do xilema secundário de Myrceugenia euosma (O. Berg) D. Legrand (Myrtaceae). Balduinia, Santa Maria, n. 16, p. 24-29, 2009b.

SANTOS, S.R. dos; MARCHIORI, J.N.C. Anatomia da madeira de Eugenia uniflora L. (Myrtaceae). Balduinia, Santa Maria, n. 17, p. 11-16, 2009c. SANTOS, S.R. dos; MARCHIORI, J.N.C. Anatomia da madeira de Myrcianthes pungens (O. Berg) D. Legrand (Myrtaceae). Balduinia, Santa Maria, n. 19, p. 25-30, 2009d.

SANTOS, S.R. dos; MARCHIORI, J.N.C.; CANTODOROW, T. S. do. Identificação botânica e anatomia da madeira de Discaria americana Gillies et Hooker. Balduinia, Santa Maria, n. 10, p. 1120, 2007a.

SANTOS, S.R. dos; MARCHIORI, J.N.C.; CANTODOROW, T.S. do; DENARDI, L. Estudo 
anatômico do lenho e descrição botânica de Rhamnus sphaerosperma Swartz (Rhamnaceae). Balduinia, Santa Maria, n. 11, p. 16-26, 2007 b. SANTOS, S.R. dos; MARCHIORI, J.N.C.; CANTODOROW, T.S. do. Estudo anatômico do lenho e descrição morfológica de Gouania ulmifolia Hooker et Arnott (Rhamnaceae). Balduinia, Santa Maria, n. 13, p. 15-26, 2008a.

SANTOS, S.R. dos; MARCHIORI, J.N.C.; CANTODOROW, T.S. do. Anatomia da madeira e descrição morfológica de Scutia buxifolia Reissek (Rhamnaceae). Balduinia, Santa Maria, n. 14, p. 19-28, 2008b.

SCHIRAREND, C. The systematic wood anatomy of the Rhamnaceae Juss. (Rhamnales) - Tribe Zizipheae. IAWA Bulletin, v.12, n. 4, p. 359-388, 1991.

SIEGLOCH, A.M.; SANTOS, S.R. dos; MARCHIORI, J.N.C. Descrição anatômica do lenho de Brunfelsia australis Bentham (Solanaceae). Balduinia, Santa Maria, n. 20, p. 16-20, 2010.

SILVA, E.A.M.; PEREIRA, L.F.R.; PINHEIRO, A.L.; RAMALHO, R.S. Variação sazonal na atividade cambial de três espécies florestais na região de Viçosa, MG. Seiva, v. 50, p. 49-52, 1991.

TEIXEIRA, L.L. Identificação botânico-dendrológica e anatomia da madeira de seis espécies euxilóforas do sudoeste paranaense. 1977. 131f. Dissertação (Mestrado em Engenharia Florestal) - Universidade federal do Paraná, Curitiba, 1977.

VLIET, G.J.C van; BAAS, P. Wood anatomy and classification of the Myrtales. Annals of the
Missouri Botanical Garden, n. 71, p. 783-800, 1984.

ZIMMERMANN, M.H. Functional xylem anatomy of Angiosperm trees. In: BAAS, P. New perspectives in wood anatomy. The Hague: Nijhoff/Junk, 1982. p. 59-70.

WEBBER, I.E., The woods of sclerophyllous and desert shrubs and desert plants of California. American Journal of Botany, v. 23, p. 181-188, 1936.

WHEELER, E.A.; BAAS, P. A survey of the fossil record for Dicotyledonous wood and its significance for evolutionary and ecological wood antomy. IAWA Bulletin, v. 12, n. 3, p. 275332, 1991.

WHEELER, E.A; BAAS, P.; RODGERS, S. Variations in dicot wood anatomy: a global analysis based on the insidewood database. IAWA Journal, v. 28, n. 3, p. 229-258, 2007.

WILKINS, A.P.; PAPASSOTIRIOU, S. Wood anatomical variation of Acacia melanoxylon in relation to latitude. IAWA Bulletin, v.10, n.2, p. 201-207, 1989.

WORBES, M. Growth rings, increment and age of trees in inundation forests, savannas and a mountain forest in the Neotropics. IAWA Bulletin, v. 10, n. 2, p. 109-122, 1989.

WOODCOCK, D.W. Occurrence of woods with a gradation in vessel size across a ring. IAWA Bulletin, v. 15, p. 377-385, 1994.

WOODCOCK, D.W.; SANTOS, G.; REYNEL, C. Wood characteristics of Amazon forest types. IAWA Journal, v. 21, n. 3, p. 277-292, 2000. 